\title{
Validación de un instrumento de estrategias para fortalecer el aprendizaje
}

\author{
Validation of an Instrument for Strategies to Strengthen Learning
}

\section{Validação de um instrumento de estratégias para fortalecer o aprendizado}

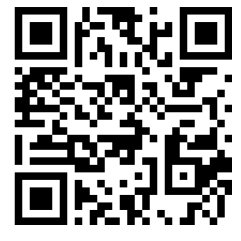

Recibido • Received • Recebido: 01 / 02 / 2018

Corregido • Revised • Revisado: 17 / 06 / 2019

Aceptado • Accepted • Aprovado: $07 / 11 / 2019$ 
doi: http://doi.org/10.15359/ree.24-1.6

URL: http://www.una.ac.cr/educare

CORREO: educare@una.cr

Resumen: El objetivo del presente trabajo fue validar un instrumento de evaluación de las estrategias que utiliza el personal docente para fortalecer el aprendizaje en el contexto de la educación inclusiva. El estudio se realizó con un enfoque cuantitativo no experimental de tipo instrumental. Participaron 210 docentes de diversas instituciones educativas. El instrumento utilizado fue el de Estrategias para fortalecer el aprendizaje, de Mitchell (2015a). Los resultados de la técnica del análisis factorial exploratorio validaron cinco subescalas relacionadas con las estrategias, las cuales son: contexto de aprendizaje, estrategias de enseñanza, estrategias del comportamiento, estrategias de cooperación interinstitucional y estrategias de recursos para el aprendizaje. Este estudio contribuye al avance del conocimiento científico sobre análisis de propiedades psicométricas de instrumentos y servirá como referencia en estudios posteriores que busquen evaluar las estrategias de enseñanza de docentes.

Palabras clave: Educación inclusiva; estrategias; docentes, validación.

Abstract: The purpose of this study was to validate an instrument aimed to evaluate the strategies that teachers use to strengthen learning. The study was conduced with a quantitative, nonexperimental, instrumental type approach. In the study, 210 teachers participated and they were from different educational institutions. The instrument used was "Strategies to Strengthen Learning" of Mitchell (2015a). The results of the exploratory factor analysis technique validated five subscales related to the strategies, which are the following: learning context, teaching strategies, behavioral strategies, interinstitutional cooperation strategies and resource strategies for learning. This study contributes to the advancement of scientific knowledge on the analysis of psychometric properties of instruments, and will serve as a reference in subsequent studies that seek to evaluate teacher's teaching strategies.

Keywords: Inclusive education; learning strategies; teachers; validation.

Resumo: O objetivo do presente trabalho é validar um instrumento de avaliação de estratégias utilizadas pelo pessoal docente para fortalecer o aprendizado no contexto da educação inclusiva. O estúdio foi realizado com um enfoque quantitativo não experimental tipo instrumental. Participaram 210 docentes de diversas instituições educativas. O instrumento utilizado foi o de Estratégias de fortalecimento do aprendizado, de Mitchell (2015a). Os resultados da técnica de análise fatorial exploratória validaram cinco escalas relacionadas com estratégias, que são: contexto de aprendizagem, estratégias de ensino, estratégias de comportamento, estratégias de cooperação interinstitucional e estratégias de recursos para o aprendizado. Esta pesquisa contribui com a avanço do conhecimento científico sobre análise de propriedades psicométricas de instrumentos e serve como referência em estudos posteriores que busquem avaliar estratégias de formação docente.

Palavras chave: Educação inclusiva; estratégias; docentes, validação.

\section{Introducción}

Uno de los temas principales en la agenday políticas educativas es el incremento de la calidad en la educación, se considera imprescindible brindar a todo el alumnado la oportunidad de contar con una educación de calidad. A diferencia de años anteriores, se habla actualmente de atención a la diversidad, aula diversificada y currículo para la diversidad, lo cual resalta la importancia de la 
práctica educativa en la que se considere la diversidad dentro del contexto escolar.

De ahí que, en la actualidad, se señala que los sistemas educativos se enfrentan al reto de brindar una educación de calidad en igualdad de oportunidades. Duk y Loren (2010) mencionan que la calidad de la educación ha sido uno de las principales aspiraciones de la política educativa y el objetivo de las reformas educativas que se han llevado a cabo en Latinoamérica.

En México, el sistema educativo nacional tiene el desafío de mejorar la calidad de la educación como lo establece el artículo $3^{\circ}$ constitucional, y esto en relación con los "materiales y métodos educativos, la organización escolar, la infraestructura educativa, y ... los docentes y ... directivos [que] garanticen el máximo logro de aprendizaje de los [estudiantes]" (Secretaría de Educación Pública [SEP], 2017, p. 43-44).

El Modelo Educativo 2017 propone hacer efectivo el derecho a la educación para todas las personas a partir de los principios que sustentan a la educación inclusiva, la cual se orienta a responder a la diversidad. En dicho modelo se mencionan la inclusión y la equidad como principios básicos para garantizar la igualdad de oportunidades, así como para promover una educación en los derechos humanos que propicie el desarrollo integral del estudiantado (SEP, 2017).

Con respecto a la inclusión, la UNESCO (2009) la considera como un proceso que permite tener en cuenta las necesidades de la diversidad del estudiantado mediante una mayor participación en el aprendizaje, y en las actividades culturales y comunitarias, lo cual implica modificaciones en los contenidos, enfoques, estructuras y estrategias.

Casanova (2011) menciona la necesidad de personalizar en todo lo posible los procesos educativos que se producen en los centros escolares. De acuerdo con esta autora, la personalización puede ser realizada a partir de dos perspectivas: la primera desde el sistema educativo general, en la que se deben considerar las exigencias de un planteamiento diversificado en relación con la estructura del sistema, la formación del profesorado, instalaciones y recursos materiales apropiados; y la segunda, en la cual el centro escolar en conjunto con la comunidad educativa considere el planteamiento anterior, el cual deberá implementar las mejores opciones para llevar a la práctica la educación inclusiva. La autora considera que la personalización de la educación y la enseñanza es la clave de la educación inclusiva.

\section{Educación inclusiva}

La educación inclusiva constituye un proceso cuyo antecedente inmediato es la integración educativa. Ambos procesos se relacionan con los modelos interactivo y social de atención a la discapacidad, aunque en el caso de la educación inclusiva se trasciende la discapacidad, pues su público destinatario es toda persona, de manera especial las niñas y los niños que viven en condición de vulnerabilidad. 
doi: http://doi.org/10.15359/ree.24-1.6

URL: http://www.una.ac.cr/educare

CORREO: educare@una.cr

En relación con los modelos mencionados, para su mejor comprensión se tiene que señalar que un modelo anterior, el modelo médico, postula que la discapacidad es una característica inherente al sujeto, por lo que la intervención que debe realizarse para compensarla debe dirigirse justamente al sujeto. En este modelo, las intervenciones siguen la siguiente lógica: se debe identificar la deficiencia del sujeto (no sus fortalezas), se debe prescribir una intervención, y esta última opera mejor cuando se realiza juntando al sujeto con otros sujetos que comparten su condición. De aquí el nacimiento de las escuelas de educación especial (García, 2015).

Por otra parte, el modelo interactivo postula que la discapacidad aparece cuando el sujeto no puede realizar alguna función o actividad debido a que tiene una condición deficitaria y cuando, además, el medio no le proporciona los apoyos que requiere para realizar dicha función (Anastasiou \& Kauffman, 2013). Si el medio proporcionara los apoyos que el sujeto necesita, no aparecería la discapacidad. Este modelo está como sustrato de la integración educativa. En este último proceso, lo que se busca es identificar los apoyos que necesitan las niñas y los niños con discapacidad (llamados necesidades educativas especiales, NEE) para potenciar sus aprendizajes $y$, entonces, que pueda cursar su trayectoria educativa con sus pares, en escuelas y aulas regulares. La lógica que se sigue es identificar sus necesidades de apoyo y sus fortalezas, proporcionarle a cada estudiante los apoyos que requiere e integrarle. Como se podrá apreciar, en muchos casos estos apoyos son individuales y específicos (por ejemplo cuando se ofrecen prótesis, tecnología para ver o escuchar mejor o para desplazarse con más facilidad y comodidad, o bien cuando se reducen los contenidos que debe aprender o se reduce su complejidad). Sin embargo, en ocasiones estos apoyos benefician a la totalidad, por ejemplo, las rampas o la diversificación de estrategias de enseñanza. En cualquier caso, la idea principal es dotarles, individualmente, los apoyos que necesitan.

Finalmente, el modelo social postula que no hay personas con discapacidad, que lo que hay son sociedades discapacitantes (Anastasiou y Kauffman, 2013). La lógica que se sigue es que no hay que identificar al sujeto como necesitado de apoyos individuales, pues este simple proceso conduce a actitudes de discriminación y a su eventual segregación. Lo que se necesita es eliminar los obstáculos que enfrentan los grupos en su medio, esto es, las barreras para el aprendizaje y la participación, y con ello potenciar sus aprendizajes. En esta perspectiva, todo el alumnado debe estudiar en las escuelas y aulas regulares y no se ofrecen apoyos específicos. A esta manera de concebir la educación inclusiva se le ha llamado educación inclusiva universal (Florian, 2010).

El riesgo que se corre con la integración educativa es que los apoyos individuales no beneficien a todo el alumnado. El riesgo que se corre con la educación inclusiva es que se invisibilice a los sujetos que necesitan apoyos específicos, individuales.

Frente a la anterior situación, Dyson (2001), quien es uno de los autores más renombrados en el terreno de la educación inclusiva (junto con Mel Ainscow y Tony Booth) propuso lo que llamó educación inclusiva responsable (otros estudios hablan de educación inclusiva moderada, entre estos, los de García y Romero, 2016). La educación inclusiva responsable postula que se 
deben ofrecer los apoyos individuales que precisan algunos grupos estudiantiles $y$, al mismo tiempo, hacer un esfuerzo por ofrecer una educación de calidad para cada quien y la totalidad.

La educación inclusiva se basa en algunos principios: aprendizaje cooperativo, aprecio y valoración de las diferencias, clima de aula favorable, expectativas docentes altas con respecto al aprendizaje de todo el alumnado y una preocupación genuina por parte del personal docente por los aprendizajes de todos sus estudiantes, entre otros. Otro principio importante se relaciona con las estrategias de enseñanza utilizadas por el personal docente.

\section{Estrategias de enseñanza}

La enseñanza es una actividad compleja e interactiva en la cual se da un proceso de comunicación y de formación. Fernández (2014) menciona que es un conjunto de roles que desarrolla el profesorado en interacción con sus estudiantes para crear oportunidades que les permitan el desarrollo de competencias. Por su parte, Blanco (2000) considera que la planeación y organización de la actividad didáctica debe hacerse de tal forma que se brinden las oportunidades de aprendizaje para el estudiantado.

Por su parte, Fernández (2014) considera que la enseñanza requiere crear un clima de confianza motivador, así como proveer los medios necesarios para que el alumnado despliegue sus potencialidades. Fernández (2014) considera que en el aprendizaje se relacionan los conocimientos adquiridos con las experiencias previas.

El mismo autor, Fernández (2014), menciona sobre la diversidad de estudiantes que pueden provenir de diferentes culturas, con distintos estilos de aprendizaje, así como también pueden tener diferentes intereses, por lo cual considera utilizar una variedad de estrategias de enseñanza. Fernández (2014) menciona algunas de las estrategias: impulsar la participación del estudiantado, motivar, partir de los conocimientos existentes y establecer relaciones entre los conocimientos previos y los nuevos, la significatividad en el aprendizaje, el andamiaje, utilizar técnicas de trabajo individual y de grupo, utilizar el aprendizaje cooperativo, usar y adaptar la tecnología, los materiales y recursos variados, las estrategias con respecto al clima de clase y el establecimiento de límites, entre otras.

Beltrán (2007) menciona que son diversas las funciones que cumplen las estrategias en relación con el aprendizaje, y menciona que las estrategias de aprendizaje favorecen y condicionan el aprendizaje significativo. Al estar relacionadas las estrategias con el aprendizaje, es posible identificar el rendimiento escolar y con ello las estrategias que pueden ser más eficaces para optimizar el aprendizaje del estudiantado. Además, menciona que las estrategias promueven un aprendizaje autónomo e independiente, de tal forma que el estudiantado pueda ser capaz de planificar, regular y evaluar su aprendizaje y dominar las estrategias de aprendizaje 
doi: http://doi.org/10.15359/ree.24-1.6

URL: http://www.una.ac.cr/educare

CORREO: educare@una.cr

llamadas metacognitivas.

Con respecto a las clases de estrategias, Beltrán (2007), menciona:

Existen diversas clasificaciones y considera que se pueden dividir teniendo en cuenta dos criterios relacionados con su naturaleza y su función. Por su naturaleza, señala a las estrategias cognitivas, metacognitivas y de apoyo; por función, ubica a las estrategias de acuerdo con los procesos a los que sirven: sensibilización, atención, adquisición, personalización (desarrollo del pensamiento crítico y creativo) recuperación y evaluación. (p. 689)

Diversos estudios han buscado evaluar las estrategias de enseñanza del profesorado a través de diferentes cuestionarios. Acosta y García (2012) aplicaron un cuestionario a docentes de universidades públicas para identificar estrategias de enseñanza en tres dimensiones: estrategias pre-instruccionales, estrategias co-instruccionales y estrategias post-instruccionales, las cuales, en la investigación, obtuvieron una Alpha de Cronbach de .95 que evidencia un cuestionario confiable. De igual manera, Acosta y Boscán (2014) evaluaron las estrategias conductistas, cognoscitivas y constructivistas de docentes de biología, y obtuvieron una consistencia interna de .88 , lo cual indica confiabilidad en la aplicación de este cuestionario.

Por su parte, Méndez y González (2011) realizaron el diseño y la evaluación de las propiedades psicométricas de la Escala de estrategias docentes para el aprendizaje significativo. En el estudio se llevó a cabo el diseño de la escala mencionada, la cual está compuesta por 12 subescalas y posteriormente se realizó una aplicación a 205 docentes de siete facultades de una universidad. En el análisis factorial exploratorio evidenció que la escala presenta una estructura unifactorial con índices de consistencia interna (alpha de Cronbach) adecuados.

Chiva y Ramos (2015) diseñaron la Escala de metodologías docentes en educación primaria, con el propósito de evaluar la percepción del alumnado sobre las metodologías utilizadas por sus docentes. Para revisar la validez de contenido se llevó a cabo la validación por juicio experto. Posteriormente, para la valoración de sus propiedades psicométricas se aplicó a un total de 324 estudiantes de sexto de primaria de nueve instituciones. De acuerdo con el análisis factorial, la escala quedó conformada por 65 ítems y 8 dimensiones, en las cuales se obtuvo una adecuada variabilidad en las respuestas, así como un alpha de Cronbach de .90, indicador de coherencia y consistencia.

En investigaciones relacionadas con la educación inclusiva se ha utilizado la Escala de adaptaciones de la enseñanza de Cardona (2003), la cual evalúa el grado de adaptaciones que realiza el profesorado en sus estrategias. El instrumento consta de 29 reactivos organizados en seis categorías: la primera de ellas se refiere al manejo efectivo del aula, la segunda hace relación al agrupamiento flexible, la tercera es la enseñanza adicional, la cuarta identifica la enseñanza estratégica, la quinta se refiere a las adaptaciones de las actividades y, por último, la sexta categoría es sobre la evaluación formativa (Cardona, 2003). 
Chiner (2011) utilizó una versión reducida de la Escala de adaptaciones de la enseñanza, la cual consta de 21 ítems y 4 subescalas: 1) estrategias de organización y manejo efectivo del aula; 2) estrategias de enseñanza y evaluación de aprendizajes; 3) estrategias de agrupamiento, y 4) estrategias de adaptación de actividades. Se aplicó a 336 docentes de diferentes escuelas y se obtuvo un alpha de Cronbach de .71. A diferencia de la escala original, la consistencia interna es menor, sin embargo continúa teniendo una confiabilidad adecuada.

El principal propósito del presente estudio es presentar el análisis factorial exploratorio del instrumento de Mitchell (2015a), el cual identifica las estrategias que utiliza el profesorado para fortalecer el aprendizaje.

\section{Estrategias para fortalecer el aprendizaje, de David Mitchell (2014)}

Mitchell (2015b) considera la educación como un concepto multidimensional en el que se pueden encontrar los siguientes aspectos: una visión de compromiso, acceso en las aulas de aprendizaje, evaluación del currículo, estrategias de enseñanza, apoyo, recursos y liderazgo.

Mitchell (2015b) presenta 27 estrategias organizadas en grupos. En el primer grupo, se encuentran las estrategias relacionadas con el contexto de aprendizaje, como por ejemplo: la educación inclusiva, la enseñanza en grupo cooperativo y el clima del aula. En la enseñanza de grupos colaborativos se utiliza una mezcla de actividades de grupo e individuales, en las cuales el alumnado tiene la oportunidad de trabajar con sus pares e intercambiar experiencias de aprendizaje (Mitchell, 2014). En la tutoría entre iguales, se realiza la promoción de las interacciones sociales, el ayudar y dar apoyo emocional al compañero o a la compañera.

En el segundo grupo se encuentran las estrategias cognitivas, como la autorregulación del aprendizaje, las estrategias de memoria y las cognitivo conductuales. La enseñanza de estrategias cognitivas se refiere a formas de ayudar al estudiantado a adquirir las habilidades cognitivas para organizar la información e integrar la información en sus conocimientos existentes.

El tercer grupo se centra en estrategias del comportamiento como la instrucción directa y la evaluación funcional. Dichas estrategias se centran en cómo los acontecimientos que se producen afectan el comportamiento posterior. Estos eventos se conocen como antecedentes y consecuencias, respectivamente.

Un cuarto grupo examina las estrategias para toda la escuela y la cooperación interinstitucional. También se consideran estrategias tales como la evaluación formativa y de retroalimentación y de tecnología de apoyo y oportunidades para el aprendizaje. Esta estrategia se refiere a las demandas de tareas externas y a las respuestas externas en el modelo de aprendizaje y enseñanza, y se relaciona con el enfoque cognitivo/ constructivista del aprendizaje.

El instrumento de estrategias para fortalecer el aprendizaje de Mitchell (2015a) consta 
doi: http://doi.org/10.15359/ree.24-1.6

URL: http://www.una.ac.cr/educare

CORREO: educare@una.cr

de estrategias cognitivas, de desarrollo de habilidades y de evaluación, también incluye aspectos relacionados con el contexto de aprendizaje y considera aspectos relacionados con la educación inclusiva.

En este sentido, el objetivo del presente trabajo es presentar la validación del instrumento Estrategias para favorecer el aprendizaje aplicadas por profesorado de diferentes instituciones educativas de una localidad del noroeste de México.

\section{Método}

\section{Participantes}

Participaron 210 docentes de diferentes instituciones educativas públicas y privadas del noroeste del país. La elección de las instituciones que participaron se llevó a cabo de manera intencional: se invitó a las escuelas a participar y a partir de ello se trabajó con el profesorado. El $23 \%$ del profesorado es de nivel preescolar, $66 \%$ de primaria, $7 \%$ de secundaria, $1 \%$ de media superior, $1 \%$ de superior. El mayor porcentaje fue de profesorado de primaria pública estatal con $39.5 \%$, el $31 \%$ de escuela pública federal, de esta misma forma el $28 \%$ eran de escuelas privadas y solo el $1 \%$ reportaba ser de otro sistema. Con respecto a la escolaridad del profesorado, el $54 \%$ tiene licenciatura o normal superior como máximo nivel de estudios, mientras que el $31 \%$ cuenta con maestría, $11 \%$ tiene normal básica sin licenciatura, solo $1 \%$ cuenta con doctorado, y el $3 \%$ no especificó. La experiencia del profesorado fue de 1 a 35 años.

\section{Instrumento}

Se utilizó el Cuestionario de estrategias para fortalecer el aprendizaje: Cuestionario para el profesor, de Mitchell (2015a), traducido y adaptado en 2015 por Fletcher, García-Cedillo y Romero-Contreras. El instrumento comprende 7 preguntas de opción múltiple, solicita datos sociodemográficos y 34 reactivos de escala tipo Likert con cuatro opciones de respuesta: muy a menudo (3), a menudo (2), pocas veces (1) y nunca (0). El tiempo de aplicación fue de 20 minutos.

\section{Procedimiento}

Se solicitó autorización en las instituciones educativas para invitar al profesorado a participar; posteriormente se solicitó el apoyo y colaboración al profesorado para responder el cuestionario. Antes de la aplicación, se le comentó al personal docente sobre la confidencialidad de la información y se optó por el consentimiento informado de manera verbal. Una vez que se realizó la aplicación, se procedió a la captura y análisis de los datos a través del programa estadístico SPSS 21 para realizar los análisis correspondientes.

\section{Resultados}

En el presente apartado se presentan los resultados de la investigación relacionados con la 
validez y confiabilidad del instrumento; cabe mencionar que los reactivos estaban organizados en cinco tipos de estrategias, por lo que el análisis factorial fue realizado por cada una de las subescalas: contexto de aprendizaje, estrategia de enseñanza, estrategia del comportamiento, estrategia de cooperación interinstitucional y recursos para el aprendizaje.

Para determinar la validez de constructo se analizó la estructura interna de cada una de las subescalas a través de la técnica multivariante de análisis factorial con el método de máxima verosimilitud con rotación Varimax, que solicita un peso factorial mayor a .40.

Para la confiabilidad del instrumento se utilizó el índice de consistencia interna de alpha

Tabla 1: Subescala: Contexto de aprendizaje

\begin{tabular}{|c|c|c|c|}
\hline No. & Reactivos & Pesos factoriales & Alpha \\
\hline 24 & Trato de crear un ambiente en el aula que sea seguro, predecible y motivador. & .932 & .948 \\
\hline 26 & $\begin{array}{l}\text { Las autoridades de mi escuela están comprometidas con la filosofía de la } \\
\text { educación inclusiva y están dispuestas a implementarla. }\end{array}$ & .908 & .949 \\
\hline 29 & $\begin{array}{l}\text { Adapto los métodos de evaluación para ajustarlos a las necesidades de todos mis } \\
\text { estudiantes. }\end{array}$ & .901 & .950 \\
\hline 30 & $\begin{array}{l}\text { Mi escuela reconoce el derecho del alumnado con necesidades educativas } \\
\text { especiales a ser educado en los salones regulares y a recibir los apoyos y recursos } \\
\text { que requieren para su desarrollo. }\end{array}$ & .898 & .950 \\
\hline 22 & $\begin{array}{l}\text { Mi escuela tiene un fuerte compromiso con la aceptación y aprecian la diversidad, } \\
\text { es sensible a las cuestiones culturales, y establece estándares altos, pero realistas. }\end{array}$ & .885 & .950 \\
\hline 27 & $\begin{array}{l}\text { El alumnado con necesidades educativas especiales que vive en la comunidad a } \\
\text { la que pertenece mi escuela asiste a esta escuela y está en los grupos apropiados } \\
\text { para su edad. }\end{array}$ & .836 & .952 \\
\hline 1 & $\begin{array}{l}\text { Para enseñar al grupo combino niñas y niños con distintos niveles de habilidad } \\
\text { para que hagan trabajo colaborativo }\end{array}$ & .829 & .953 \\
\hline 5 & $\begin{array}{l}\text { Propicio activamente la participación de los padres y madres en el desarrollo } \\
\text { académico de sus hijos e hijas. }\end{array}$ & .819 & .953 \\
\hline 4 & $\begin{array}{l}\text { Coopero de forma activa con otros grupos profesionales y paraprofesionales (ej. } \\
\text { auxiliares). }\end{array}$ & .738 & .956 \\
\hline 19 & $\begin{array}{l}\text { Las características físicas de mi salón (por ejemplo, la iluminación, la ventilación, } \\
\text { el sonido) son óptimas para el aprendizaje. }\end{array}$ & .720 & .956 \\
\hline 2 & Uso la tutoría entre pares (compañeros y compañeras de clase). & 686 & .957 \\
\hline
\end{tabular}

Nota: Elaboración propia.

de Cronbach.

La primera subescala que se refiere a contexto de aprendizaje, está compuesta por 11 
doi: http://doi.org/10.15359/ree.24-1.6

URL: http://www.una.ac.cr/educare

CORREO: educare@una.cr

Tabla 2: Subescala: Estrategias de enseñanza

\begin{tabular}{|c|c|c|c|}
\hline No. & Reactivos & Pesos factoriales & Alpha \\
\hline 25 & $\begin{array}{l}\text { Animo a mis estudiantes a establecer sus propias metas y evaluar } \\
\text { continuamente su progreso para alcanzarlas. }\end{array}$ & .892 & .922 \\
\hline 7 & $\begin{array}{l}\text { De manera cotidiana, ayudo a mis estudiantes a definir sus propias metas, } \\
\text { a monitorear su comportamiento, a elaborar planes de acción personal, a } \\
\text { autoevaluar su progreso respecto del logro de sus objetivos, y a ajustar sus } \\
\text { metas y sus planes de acción. }\end{array}$ & .863 & .926 \\
\hline 6 & $\begin{array}{l}\text { Les enseño explícitamente a mis estudiantes estrategias cognitivas. Ej: las } \\
\text { estrategias de solución en problemas. }\end{array}$ & .861 & .926 \\
\hline 10 & $\begin{array}{l}\text { Les ayudo a mis estudiantes a mejorar su comprensión lectora mediante la } \\
\text { predicción, el cuestionamiento y el resumen de textos. }\end{array}$ & .861 & .926 \\
\hline 9 & $\begin{array}{l}\text { En mis clases, yo regularmente reviso y practico las principales ideas de las } \\
\text { lecciones anteriores. }\end{array}$ & .852 & .927 \\
\hline 14 & $\begin{array}{l}\text { Utilizo estrategias de enseñanza directa, explícita y sistemática basada en } \\
\text { planes de lecciones con guías detalladas, y espero altos niveles de éxito ( } 90 \\
\text { por ciento) y realizo evaluaciones frecuentes. }\end{array}$ & .852 & .927 \\
\hline 8 & $\begin{array}{l}\text { Les enseño explícitamente a mis estudiantes una variedad de estrategias } \\
\text { para mejorar su memoria. }\end{array}$ & .786 & .934 \\
\hline
\end{tabular}

Nota: Elaboración propia.

reactivos y posee una varianza total explicada de $69.87 \%$, el peso factorial de los reactivos oscila entre .93 y .68. Se obtuvo una confiabilidad de alfa de Cronbach de .956 (ver Tabla 1).

Tabla 3: Subescala: Estrategias del comportamiento

\begin{tabular}{clcc}
\hline No. & \multicolumn{1}{c}{ Reactivos } & Pesos factoriales & Alpha \\
\hline 11 & $\begin{array}{l}\text { Utilizo estrategias conductuales en mi enseñanza, sobre todo en el caso del } \\
\text { alumnado con dificultades en el comportamiento. }\end{array}$ & .923 & .847 \\
$12 \begin{array}{l}\text { Cuando algún alumno o alguna alumna presenta conductas sociales } \\
\text { indeseables, me dedico a analizar qué funciones o propósitos podrán } \\
\text { cumplir estas conductas y diseño estrategias para modificarlas. }\end{array}$ & .901 & .864 \\
$3 \quad \begin{array}{l}\text { Enseño a mis estudiantes habilidades sociales adecuadas. } \\
23\end{array}$ & $\begin{array}{l}\text { Mi escuela tiene programas de apoyo conductual positivo que enfatizan la } \\
\text { prevención y la reducción de problemas de conducta crónicos, la enseñanza } \\
\text { activa de habilidades adaptativas, un continuo de consecuencias para los } \\
\text { problemas de conducta e intervención. }\end{array}$ & .894 & .860 \\
\hline
\end{tabular}

Nota: Elaboración propia.

\begin{tabular}{c}
\hline 10 Dora Yolanda Ramos-Estrada, Ismael García-Cedillo, Mirsha Alicia Sotelo-Castillo, Mercedes Idania López-Valenzuela y Luisa Dolores Murillo-Parra \\
Los artículos de la Revista Electrónica Educare del Centro de Investigación y Docencia en Educación de la Universidad Nacional, Costa Rica, se comparten bajo términos de la Licencia \\
Creative Commons: Reconocimiento, No Comercial, Sin Obra Derivada 3.0 Costa Rica. Las autorizaciones adicionales a las aquí delimitadas se pueden obtener en el correo: educare@una.cr
\end{tabular} 
En la Tabla 2 se reporta la subescala de estrategias de enseñanza, está compuesta por siete reactivos y posee una varianza total explicada de $72.75 \%$, los pesos factoriales de los reactivos oscilan entre .89 y .85. Se obtuvo una confiabilidad de alfa de Cronbach de .931 (ver tabla 2).

Tabla 4: Subescala: Estrategias de cooperación interinstitucional

\begin{tabular}{|c|c|c|c|}
\hline No. & Reactivos & $\begin{array}{c}\text { Pesos } \\
\text { factoriales }\end{array}$ & Alpha \\
\hline 34 & $\begin{array}{l}\text { Trato de asegurarme de que mis estudiantes tengan suficiente tiempo para } \\
\text { aprender. }\end{array}$ & .925 & .923 \\
\hline 13 & $\begin{array}{l}\text { Cuando mis estudiantes muestran percepciones negativas de sí, les ayudo a } \\
\text { modificarlas y si estos problemas son graves o prolongados los canalizo para } \\
\text { que reciban ayuda profesional. }\end{array}$ & .896 & .926 \\
\hline 15 & $\begin{array}{l}\text { Evalúo el progreso de todo el alumnado durante las clases y ajusto mi } \\
\text { enseñanza de acuerdo con el resultado de la evaluación. }\end{array}$ & .895 & .926 \\
\hline 28 & $\begin{array}{l}\text { Adapto los métodos del currículo para satisfacer las necesidades de la } \\
\text { totalidad de mis estudiantes. }\end{array}$ & .884 & .927 \\
\hline 16 & Proporciono retroalimentación a todo el alumnado en mi clase. & .883 & .927 \\
\hline 21 & $\begin{array}{l}\text { Mi escuela hace seguimiento del progreso de todo el alumnado, identifica a } \\
\text { aquel cuyo logro está muy por debajo del de sus pares y toma decisiones sobre la } \\
\text { intensidad y la probable duración de las intervenciones basadas en la respuesta } \\
\text { del alumnado a la institución a través de múltiples niveles de intervención. }\end{array}$ & .857 & .930 \\
\hline 20 & $\begin{array}{l}\text { Proporciono a mis estudiantes una amplia variedad de medios de } \\
\text { representación (visuales, auditivos, táctiles), de acción, expresión y de } \\
\text { participación. }\end{array}$ & .820 & .933 \\
\hline 18 & $\begin{array}{l}\text { En el caso de los lectores o lectoras principiantes, enseño procesos fonológicos } \\
\text { (por ejemplo, las habilidades de escucha y conciencia fonológica). }\end{array}$ & .498 & .953 \\
\hline
\end{tabular}

Nota: Elaboración propia.

La tercera subescala corresponde a la estrategias del comportamiento, está compuesta por 4 reactivos que poseen una varianza total explicada de $77.61 \%$, el peso factorial de los reactivos oscila entre .92 y .80. Se obtuvo una confiabilidad de alfa de Cronbach de .902 (ver Tabla 3). 
doi: http://doi.org/10.15359/ree.24-1.6

URL: http://www.una.ac.cr/educare

CORREO: educare@una.cr

Tabla 5: Subescala: Estrategia de recursos para el aprendizaje

\begin{tabular}{clcc}
\hline No. & \multicolumn{1}{c}{ Reactivos } & Pesos factoriales & Alpha \\
\hline 32 & $\begin{array}{l}\text { Los maestros y las maestras de mi escuela reciben los apoyos profesionales } \\
\text { necesarios y apropiados de parte de del personal consultor especialista, } \\
\text { terapeutas y otros grupos profesionales, así como de auxiliares de la } \\
\text { educación y otros servicios paraprofesionales. }\end{array}$ & .864 & .544 \\
$33 \quad \begin{array}{l}\text { Mi escuela ha sido dotada del equipo necesario y apropiado y del personal } \\
\text { que se necesita para trabajar con estudiantes con necesidades educativas } \\
\text { especiales. }\end{array}$ & .798 & .616 \\
$31 \quad \begin{array}{l}\text { Mi escuela cuenta con accesos adecuados a los salones de clase y dentro } \\
\text { de estos para personas con discapacidad. }\end{array}$ & .723 & .657 \\
$17 \quad \begin{array}{l}\text { Utilizo las tecnologías de la información y de la comunicación disponible, } \\
\text { incluyendo la tecnología compensatoria (aparatos auditivos, macrotextos, } \\
\text { etc.) para el alumnado con discapacidad. }\end{array}$ & .535 & .749 \\
\hline
\end{tabular}

Nota: Elaboración propia.

En la Tabla 4 se presentan los resultados de la subescala de estrategia de cooperación interinstitucional, la cual está compuesta por 8 reactivos y posee una varianza total explicada de $70.93 \%$, donde los pesos factoriales de los reactivos oscilan entre .92 y .49 de esta misma forma se obtuvo una confiabilidad de alfa de Cronbach de .931 (ver Tabla 4).

La quinta subescala que corresponde a la estrategia de recursos para el aprendizaje está compuesta por 4 reactivos que tienen una varianza total explicada de $54.78 \%$, el peso factorial de los reactivos oscila entre .86 y .53 y una confiabilidad de alfa de Cronbach de .709 (ver Tabla 5).

\section{Discusión y conclusiones}

Actualmente se puede encontrar información y trabajo realizado en educación inclusiva, así como las aportaciones que se están realizando en el área. En este sentido, se considera importante contar con estudios que exploren las propiedades psicométricas de los instrumentos que se desarrollen para contar con índices de confiabilidad y validez adecuados que permitan que la generación de conocimiento en el tema tenga fundamentos sólidos.

En el presente trabajo se identificó que en el instrumento de Estrategias para fortalecer el aprendizaje, que en la versión original (Mitchell, 2015a) se presentó con la organización de los reactivos en cuatro grupos; al llevar a cabo la validación del instrumento en población sonorense, los ítems se agruparon en cinco subescalas con pesos factoriales adecuados. Cabe señalar que la agrupación de los reactivos es similar a la de la escala original. 
De igual manera, se puede observar que las estrategias de enseñanza, el apoyo entre profesionales y la importancia de los recursos en las instituciones continúa teniendo predominancia, pero debe agregarse una quinta subescala, denominada cooperación interinstitucional, la cual hace referencia al apoyo que hay dentro de la institución para contribuir al aprendizaje del estudiantado.

Es importante destacar que este estudio contribuye a la producción de escalas destinadas a evaluar las estrategias de enseñanza que utiliza el profesorado en su labor docente, ya que, de acuerdo con la revisión de la bibliografía, son escasas las investigaciones en las que se han utilizado escalas reconocidas y confiables (Cardona, 2003; Chiner, 2011; Chiva y Ramos, 2015).

Los instrumentos que valoran las estrategias de enseñanza utilizadas por el profesorado que implementa procesos de inclusión en sus aulas y escuelas deben utilizarse para"reflexionar sobre su propia práctica, enfocándose en los aspectos de sus prácticas que tienen más desarrollados para lograr los objetivos de la inclusión e identificando los que han desarrollado menos" (García, Romero, Escalante y Flores, 2018, p. 20). Por esta razón no proporcionan normas de calificación.

A partir de este trabajo, se sugiere que las próximas investigaciones deben ir encaminadas a la replicación del análisis, pero con una muestra más grande de participantes, en donde se pueda observar la representatividad de la población mexicana. Esto va de la mano con la promoción del uso de este tipo de instrumentos para evaluar aspectos esenciales en la docencia, incluyendo tanto a profesorado en servicio como a docentes en formación.

Además, no hay que dejar de lado que, en la actualidad, con el movimiento de educación inclusiva, las estrategias deben ir enfocadas a la atención de la diversidad, lo cual implica la inclusión de la identificación de los principales estilos y ritmos de aprendizaje del estudiantado, el fomento de valores, las adecuaciones curriculares y la promoción del trabajo colaborativo (Fernández, 2013). Por lo tanto, es necesario avanzar en estudios que garanticen claramente el trabajo en el movimiento inclusivo para impulsar, de esta manera, la mejora en los procesos de enseñanza aprendizaje y una educación de calidad en México.

\section{Agradecimientos}

Agradecimiento al Programa de Fomento y Apoyo a Proyectos de Investigación (PROFAPI 2016). Número de folio del proyecto 2016-0092.

\section{Referencias}


doi: http://doi.org/10.15359/ree.24-1.6

URL: http://www.una.ac.cr/educare

CORREO: educare@una.cr

Acosta, S. y Boscán, A. (2014). Estrategias de enseñanza para promover el aprendizaje significativo de la biología en la Escuela de Educación, Universidad del Zulia. Multiciencias, 14(1), 67-73. Recuperado de http://www.redalyc.org/articulo.oa?id=90430816010

Acosta, S. y García, M. C. (2012). Estrategias de enseñanza utilizadas por los docentes de biología en las universidades públicas. Omnia, 18(2), 67-82. Recuperado de http://www.redalyc. org/articulo.oa?id=73723402005

Anastasiou, D. y Kauffman, J. M. (2013). The social model of disability: Dichotomy between Impairment and disability. Journal of Medicine and Philosophy, 38, 441-459. doi: https:// doi.org/10.1093/jmp/jht026

Beltrán J. (2007). Estrategias de aprendizaje. En E. González y J. A. Bueno (Eds.), Psicología de la educación y del desarrollo en la edad escolar (pp. 679-710). Madrid: Editorial CCS.

Blanco, R. (2000). La atención a la diversidad en el aula y las adaptaciones del currículum. En Á. Marchesi, C. Coll y J. Palacios (Comps.), Desarrollo psicológico y educación (Vol. 3, pp. 411438). Madrid: Alianza.

Cardona, M. C. (2003). Inclusión y cambios en el aula vía adaptaciones instructivas. Revista de Investigación Educativa, 21(2), 465-487. Recuperado de https://rua.ua.es/dspace/ bitstream/10045/24896/1/2003 Cristina Cardona Rev Inv Edu.pdf

Casanova, M. A. (2011). Educación inclusiva: Un modelo de futuro. Barcelona: Wolters Kluwer

Chiner, E. (2011). Las percepciones y actitudes del profesorado hacia la inclusión del alumnado con necesidades educativas especiales como indicadores del uso de prácticas educativas inclusivas en el aula (Tesis doctoral), Universidad de Alicante, España. Recuperada de https://rua. ua.es/dspace/bitstream/10045/19467/1/Tesis Chiner.pdf

Chiva, I. y Ramos, G. (2015). Diseño y validación de una escala para evaluar las estrategias de gestión e intervención docente en educación primaria. Revista Actualidades Investigativas en Educación, 15(3), 1-24. doi: https://doi.org/10.15517/aie.v15i3.20927

Duk, C. y Loren, C. (2010). Flexibilización del currículum para atender la diversidad. Revista Latinoamericana de Educación Inclusiva, 4(1), 187-210. Recuperado de http://www.rinace. net/rlei/numeros/vol4-num1/art9.pdf

Dyson, A. (2001). Special needs in the twenty-first century: Where we've been and where we're going. British Journal of Special Education, 28(1), 24-29. doi: https://doi.org/10.1111/1467-8527. t01-1-00200

Fernández, J. M. (2013). Competencias docentes y educación inclusiva. Revista Electrónica de Investigación Educativa, 15(2), 82-99. Recuperado de http://redie.uabc.mx/vol15no2/ contenido-fdzbatanero.html 
Fernández, J. M. (2014). Un currículo para la diversidad. Madrid: Síntesis. Recuprado de https:// www.sintesis.com/biblioteca-de-educacion-145/un-curriculo-para-la-diversidadebook-1506.html

Florian, L. (2010). Special education in an era of inclusion: The end of special education or a new beginning? The Psychology of Education Review, 34(2), 22-29. Recuperado de https:// www.academia.edu/24487820/Special education in an era of inclusion The end of special education or a new beginning

García, I. (2015). Integración educativa o educación inclusiva, ¿qué conviene a México? Cátedra CUMEX de Psicología. En A. García, y O. Cruz, O. (Eds.), Los retos de la psicología en la sociedad contemporánea. Tuxtla Gutiérrez Chiapas, México: Universidad de Ciencias y Artes de Chiapas.

García, I. y Romero, S. (2016). Avances de la integración educativa/inclusión educativa y la formación docente para la inclusión en México. Aguascalientes, México: Centro de Estudios Jurídicos y Sociales-UASLP.

García, I., Romero, S., Escalante, L. y Flores, V. J. (2018). Algunas propiedades psicométricas de las guías para evaluar prácticas inclusivas en el aula. Revista Española de Orientación y Psicopedagogía, 29(2), 8-28. doi: https://doi.org/10.5944/reop.vol.29.num.2.2018.23150

Méndez, L. M. y González, M. T. (2011). Escala de estrategias docentes para aprendizajes significativos: Diseño y evaluación de sus propiedades psicométricas. Revista Actualidades Investigativas en Educación, 11(3), 1-39. doi: https://doi.org/10.15517/aie.v11i3.10217

Mitchell, D. (2014). What really works in Special and Inclusive Education. Using evidence-based teaching strategies. New York: Routledge. doi: https://doi.org/10.4324/9780203105313

Mitchell, D. (2015a). Strategies for enhancing learning: Teacher questionnaire [A selfrating scale]. Recuperado de https://routledgetextbooks.com/textbooks/ author/ mitchell-9780415623230/rating.php

Mitchell, D. (2015b). Inclusive Education is a Multi-faceted Concept. Center for Educational Policy Studies Journal 5(1), 9-30. Recuperado de http://www.pedocs.de/volltexte/2015/10611/ pdf/cepsj 20151 Mitchell Inclusive Education.pdf

Secretaría de Educación Pública (SEP). (2017). Modelo educativo para la educación obligatoria. Educar para la libertad y la creatividad. México: Autor. Recuperado de https://www.gob. $\mathrm{mx} / \mathrm{cms} / \mathrm{uploads} /$ attachment/file/198738/Modelo Educativo para la Educacio $\mathrm{n}$ Obligatoria.pdf

UNESCO. (2009). Direcciones sobre políticas de inclusión en la educación. París: Autor. Recuperado de http://unesdoc.unesco.org/images/0017/001778/177849s.pdf 